\title{
2 Assessing Socio-ecological Systems Using Social Media Data: 3 An Approach for Forested Landscapes in Tierra del Fuego, 4 Argentina
}

\author{
5 Alejandro Huertas Herrera ${ }^{1}$ (D) - Mónica Toro Manríquez ${ }^{2} \cdot$ Rosina Soler $^{1}$. \\ 6 Cristian Lorenzo ${ }^{1,3}$. María Vanessa Lencinas ${ }^{1}$. Guillermo Martínez Pastur ${ }^{1}$
}

7 Accepted: 25 March 2021

8 (c) The Author(s), under exclusive licence to Springer Nature B.V. 2021

\section{Abstract} The purpose of this study was to analyse how visitors valued a socio-ecological system 1 through the use of social media data. We gathered YouTube's videos of Ushuaia city and its surrounding forested landscapes (Tierra del Fuego, Argentina) posted between 2010 and 2020. We used the screen time (seconds) of each video to compare the value of visitors on biophysical, cultural, and biodiversity attributes of the studied socio-ecological system. Each of the visitors registered differently the same attribute (e.g. mountains, forests, signposts, fauna, among others), therefore we assessed the time each visitor spend on any attribute, that was calculated considering the focus and scale through which it was observed. Based on our analyses, we found a diversity of attributes with different valuation data for each visitor. Attributes were classified as biophysical, cultural, and biodiversity AQ1 variables, and the origin of the visitors (e.g. regions of the world) was also evaluated, with descriptive and multivariate analyses. Results indicated that visitors give more value to biophysical and cultural attributes compared to local biodiversity. These outputs highlight the need to explore and implement alternative methods to assess the socio-ecological values to achieve management objectives, and to include socio-ecological attributes in the study area as key indicators to create better tools and solutions for conservation issues. In this context, we provide a new insight into how visitors can appreciate different socio-ecological values.

Laboratorio de Recursos Agroforestales, Centro Austral de Investigaciones Científicas (CADICCONICET), Houssay 200 (9410), Ushuaia, Tierra del Fuego, Argentina

Departamento de Ciencias Agropecuarias Y Acuícolas, Universidad de Magallanes (UMAG),

A7 ${ }^{3}$ Instituto de Ciencias Polares, Ambiente Y Recursos Naturales (ICPA), Universidad Nacional de A8 Tierra del Fuego (UNTDF), Fuegia Basket 251 (9410), Ushuaia, Tierra del Fuego, Argentina 


\section{Introduction}

In a globalized world, the socio-ecological context is critical to assess how humans value nature (Díaz et al., 2018; Mastrangelo et al., 2015). The global scope of human activities affects the entire natural ecosystems, and for this, the new approaches considered them as socio-ecological systems (Collins et al., 2010). Visitor attitudes towards nature (e.g. nature tourism at a National Park or a city tour) allow understanding the preferences that humans (especially non-local people) have for biophysical, cultural, or biodiversity values (Kim et al., 2014; Lenormand et al., 2018; Martínez Pastur et al., 2016). However, visitors place a complex series of demands of socio-ecological concern, e.g. celebrating human achievement rather than appreciation for nature can downplay visitor perceptions of conservation reserves as tools for nature conservation (Lenormand et al., 2018; Newsome \& Hughes, 2018). How visitor appreciate a socio-ecological system can be a key to carry out actions for the conservation of natural and cultural landscapes, through which important advances are made in the understanding of different socio-ecological values (Schröter et al., 2017; Thomas-Walters et al., 2020), e.g. decision-making must carefully consider what is being promoted and allowed in conservation reserve networks (Newsome $\&$ Hughes, 2018). The increment of the public interest can lead to improve the current conservation activity by people itself (Fukano et al., 2020). Thereby, it is necessary to understand the factors that shape people's behaviour and attitudes towards nature and cultural patrimony to delineate new conservation strategies (Hodge, 1997; Rosalino et al., 2017), as well as, to enhance other types of studies related to citizen science with the purpose of developing more accurate nature conservation strategies (Schröter et al., 2017), e.g. visitors move according to personal preferences, frequently influenced by the attractiveness of a particular area (Lenormand et al., 2018). Therefore, the socio-ecological approach requires the understanding of the idiosyncrasies of local contexts (e.g. the value of local nature) (Ballari et al., 2020), which favour adaptive social ecosystem management and biodiversity preservation, such as the ecological transition of societies (Díaz et al., 2015).

A better understanding of the interactions between human and nature is critical for conservation science and for land planning (Hodge, 1997). Despite collecting relevant data remains a challenge (Toivonen et al., 2019), social networks are a rich source of content that shows human attitudes and participatory culture (Burgess \& Green, 2018; Kim et al., 2014). People currently spend billion hours per day watching video contents on the web and social media platforms (Tong et al., 2020). Consequently, the information that can be obtained from social networks becomes significant to understand how humans value nature (Beneito-Montagut, 2011; Toivonen et al., 2019). This fact can be explained due to in part the users online tend to select the information that supports and adhere to their beliefs (Bessi et al., 2016). The social networks as YouTube (Alphabet Inc., United States) offer access to diverse cultural products and perspectives around the world (e.g. cultural values), allowing theories to be tested that the web facilitates global cultural convergence (Park et al., 2017). In particular, YouTube has a great potential to understand socio-ecological concerns analysing video contents related to recreational activities and social engagement of the viewers (Otsuka \& Yamakoshi, 2020).

The visitor priorities and consumption patterns evolve every day, and people travel more frequently and further away from home, which opens up new challenges in understanding the constraints to worldwide socio-ecological systems (Lenormand et al., 2018). Thus, regional perspectives can provide insights from the cultural patterns and global implications (Garavan et al., 2016). The World Bank Group considers the world's regions include 
economies at all income levels (www.worldbank.org), which can be used to develop indicators for analytical purposes based on the regions with areas and populations within a rough order of magnitude (Georgeson et al., 2017). This context may provide relevant information for the socio-ecological connectivity between worldwide human groupings with a particular socio-ecological system and international agencies such as The Intergovernmental Science-Policy Platform on Biodiversity and Ecosystem Services (IPBES), e.g. identification and promotion of development policy support tools and methodologies in the field of biodiversity and ecosystem functions and services.

To understand visitor patterns and how humans interact with the environment is essential for holistic approaches of socio-ecological systems, focusing on the different components and how they interact (Lenormand et al., 2018), especially in areas with low data availability such as Southern Patagonia (Martínez Pastur et al., 2016). The aim of this study was to analyse how visitors' value socio-ecological systems using social media data (YouTube) in forested landscapes of Tierra del Fuego (Argentina). Specifically, we want to answer the following questions: (i) what are the biophysical, cultural and biodiversity attributes which have a significant value for visitors?; (ii) does the valuation of each attribute varied according to the visitor origin or provenance?; and (iii) based on the previous answers, what are the implications of the studied approach for future research (e.g. ecosystem services, land use policy)? We hypothesized that the analyses of social media data (e.g. YouTube's videos) allow differentiating the appreciations of visitors from different parts of the world and making an assessment of the socio-ecological values. Here, we first described materials and methods (study area, sampling design and data taking and data analyses) and then the empirical results based on the video surveys and multivariate analyses. Then, we discussed the biophysical, cultural and biodiversity attributes, the attribute valuation according to the visitor provenance, and the implications of the studied approach for future research. Finally, we offered concluding remarks for potential uses of the proposed methodology.

\section{Materials and Methods}

\subsection{Study Area}

The studied socio-ecological system $\left(54^{\circ} 40^{\prime}-54^{\circ} 53^{\prime} \mathrm{S}, 67^{\circ} 54^{\prime}-68^{\circ} 36^{\prime} \mathrm{W}\right)$ was Ushuaia city and its surrounding forested landscapes, including the Tierra del Fuego National Park (Fig. 1). The study area covers near $1500 \mathrm{~km}^{2}$ with an elevation range from 0 to $\sim 1500$ m.a.s.l. The dominant vegetation types consist in mixed Nothofagus forests (evergreen and deciduous) and open areas like grasslands and peatlands (Toro Manríquez et al., 2019). The continuous range of highlands runs from west to east, where glaciers and peatlands play an important role in the hydrology regulation and tourism (Grootjans et al., 2010). Ushuaia city and its related attractions offer an interesting opportunity to investigate different socio-ecological issues because: (i) the study area constitutes a human and natural matrix that contrasts between urban and "pristine" natural systems; (ii) visitors may easy access to different areas without major economic restrictions due to the proximity of the natural and cultural attractions (e.g. Tierra del Fuego National Park); and (iii) this study area not contains a unique iconic tourist attraction that overshadows other values, as occur in Los Glaciares National Park in Argentina (e.g. Perito Moreno glacier) or Torres 


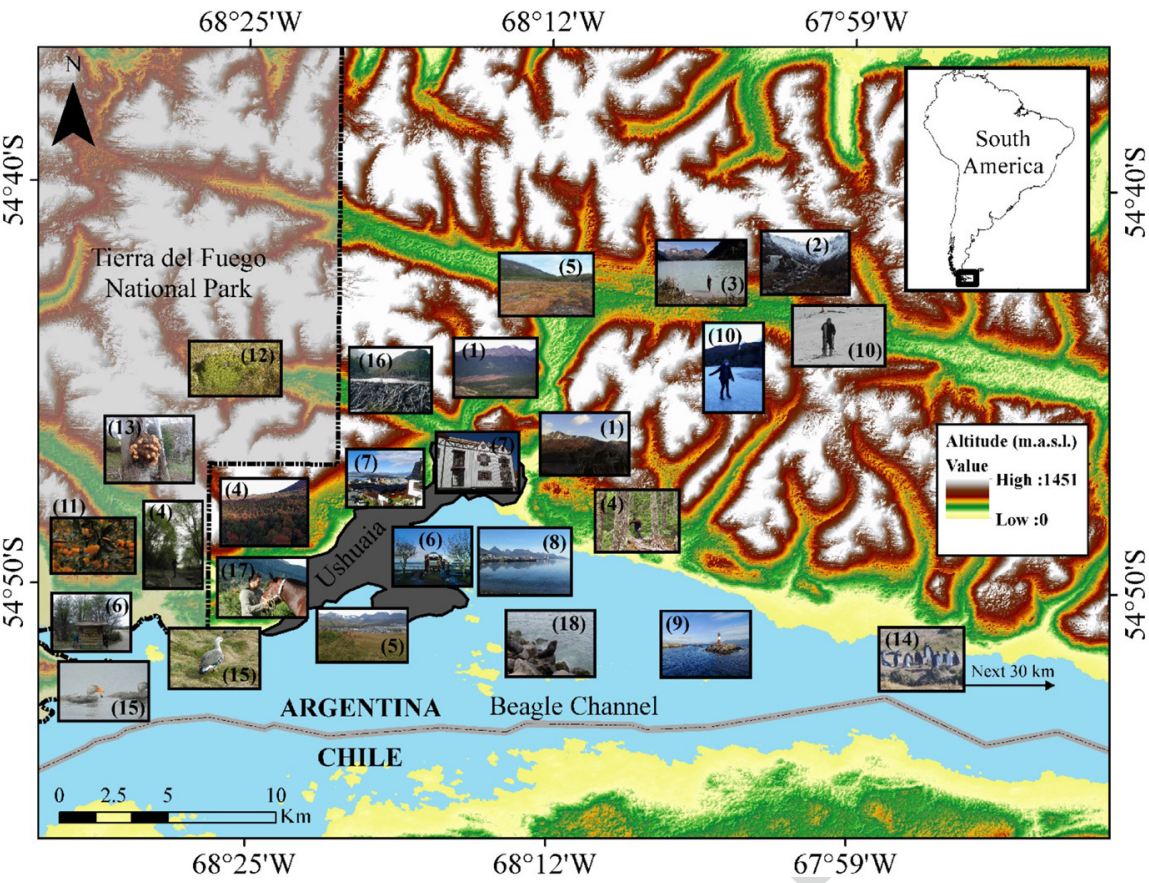

Fig. 1 Map of the Ushuaia city and its surroundings (Tierra del Fuego, Argentina), showing examples of the biophysical, cultural and biodiversity attributes of the studied socio-ecological system. Biophysical: (1) mountains, (2) high mountains, (3) water, (4) forest, (5) open land; Cultural: (6) signposts, (7) urban, (8) bay, (9) sights, (10) Ski center; and Biodiversity: (11) shrub, (12) cushion, (13) fungi, (14) penguins, (15) goose and ducks, (16) rodents, (17) odd-toed ungulate, (18) eared seals. For more details see Table1

del Paine National Park in Chile (e.g. Paine Horns). In these natural parks, visitors focus on few elements and not capture other natural diversity and city beauty elements.

\subsection{Sampling Design and Data Taking}

We explored videos posted on YouTube platform (www.youtube.com) between 2010 and 2020 by visitors from different World's regions (Fig. 2). To do this, we conducted a video search based on the keywords "Tierra del Fuego National Park" and/or "Ushuaia Tierra del Fuego" translated into 109 languages using Google Translate (www.translate.google. com) from Afrikaans to Zulu (see Appendix). For the search criteria, we used the following search filter in YouTube: Sort by-Relevance; Type-All; Upload date-Any; Duration-All. We focused on the posted videos between 5 and $10 \mathrm{~min}$ length, but shorter $(<4 \mathrm{~min})$ and longer ( $>20 \mathrm{~min}$ ) videos were also considered during a second round analyses. The videos containing content in photographs were considered as a normal video. We did not select videos focused on expeditions or famous YouTubers that promote their channels (e.g. extreme sports, food). Those videos containing images from other symbolic parts of Patagonia than Ushuaia (e.g. videos recapitulating multiple Patagonian places within a long tour) were cut, and those sections were excluded from the analyses.

We conducted a visual analysis of the content of posted videos $(n=100)$. Detailed data on the biophysical, cultural and biodiversity attributes were obtained according to each 


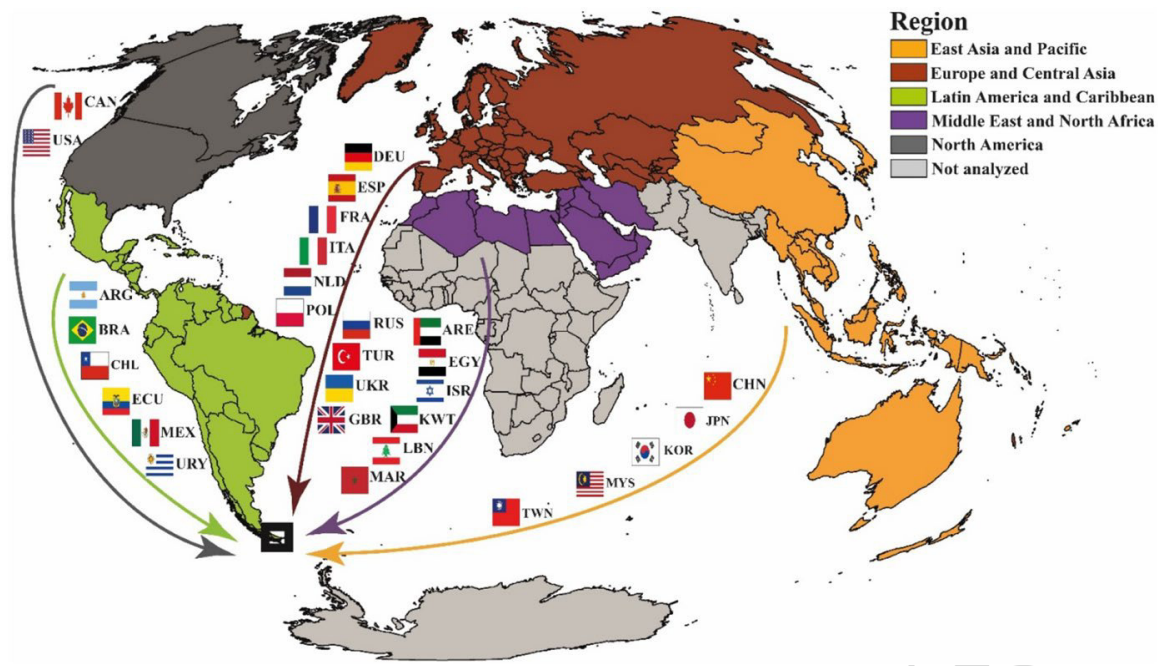

Fig. 2 Visitors from the different World's regions. The colored arrows indicate the visitor's provenance (flag's country next to the arrow) of analyzed videos. The World's regions were classified according to the World Bank analytical grouping (https://data.worldbank.org). The black square correspond to the study area. Codes in Appendix

person captured in the field and uploaded in the video. For this, we estimated the screen time (seconds) of each person filmed and/or photographed (Fig. 3). Thus, time was used as a proxy for the value or relevance that visitors give to the different attributes, e.g. they
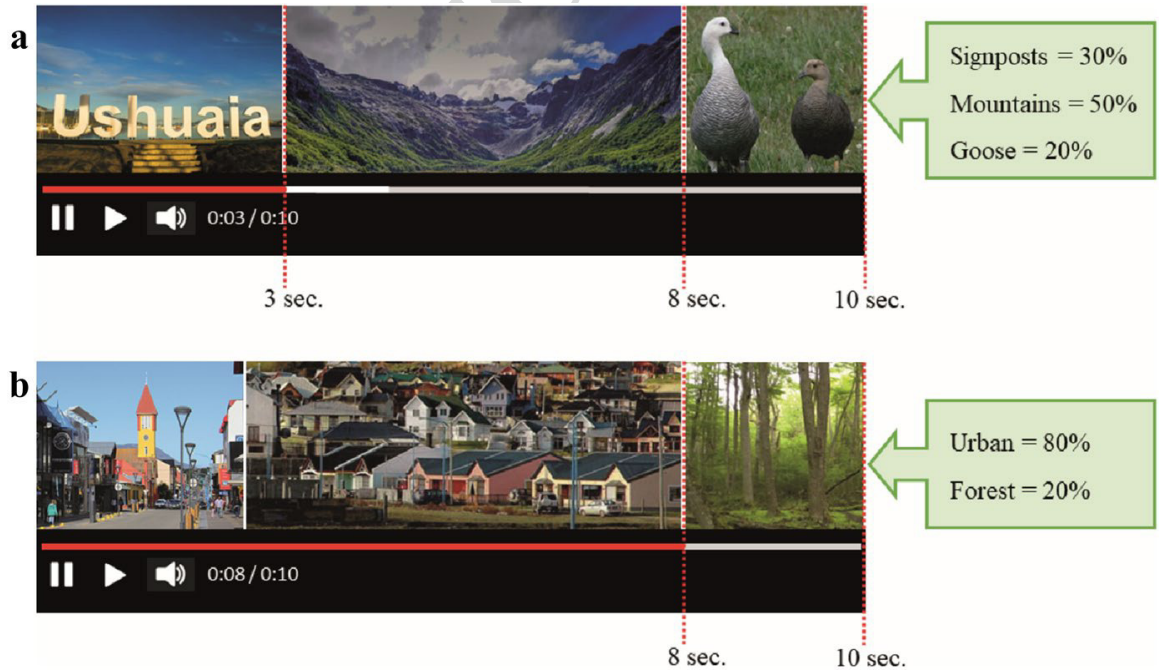

Fig. 3 Examples of the screen time of the biophysical, cultural, and biodiversity attributes observed in each video. The red line represents the screen time (seconds) spent on each captured attribute. Green box attributes computed in percentages. a an example where three attributes were observed, and $\mathbf{b}$ an example where one attribute (cultural) was more valuable than others (biodiversity) 
spend more time filming or selecting images catching the most important attributes for them, and share such experience with others. The diversity of biophysical attributes was classified as mountains, high mountains, water, forests, open-lands, and was associated to the cultural attributes including both human constructions and activities (e.g. ski sports, sailing). In addition, the diversity of attributes associated with biodiversity was classified according to the kingdom (fungi or plants), growth habit of plants (e.g. graminoid, forb, cushion, shrub, tree), and class level of animals (e.g. penguins, cormorants, goose and ducks, canids, rodents, odd-toed ungulate, eared seals, bumblebee). The relationships between the size of one observed attribute (e.g. single object or landscape) when were compared to another (e.g. the proportion occupied by the attribute on the screen), were used to choose the value for each question. This means that if the visitor focused their camera lens on a mountain landscape where forests were $20 \%$, and the high-top mountain was $80 \%$ (visual estimation of image), the assigned value was the high-top mountain; even if the camera lens captured a bird flying, water, inhabited areas, etc. Subsequently, to determine the visitor's origin country, an exhaustive exploration was made from the YouTube platform (e.g. video comments), Facebook (Facebook Inc., United States), Instagram (Facebook Inc., United States) or Google platform (Alphabet Inc., United States). Data extracted from the videos were combined with The World's region information classified according to the World Bank analytical grouping (Fig. 2). Field verifications were made between November 2019 and February 2020 to check the flora and fauna (screenshot of the species) of concurred places (e.g. Tierra del Fuego National Park) in order to have better certainty of the biodiversity observed (e.g. fungi) by the visitors in the studied socioecological system.

\subsection{Data Analyses}

Descriptive statistical analyses in terms of occurrence frequency were used to define the proportion of the sampled groups (e.g. World's regions) in which each biophysical, cultural, and biodiversity attributes occurred (e.g. natural landscapes, species). Then, screen time (seconds) calculated for the different attributes were transformed in percentage and were analyzed using a multivariate statistical methodology. Multivariate analyses were conducted to explore the relationships between World's regions and the measured attributes. To select the appropriate multivariate method, we first evaluated the response type of the data, e.g. linear or unimodal. According to ter Braak and Šmilauer (2015), a linear model is most useful when the gradient length is shorter than 3 standard deviations (SD), whereas a unimodal model is a better choice when it is larger than 4 SD. For intermediate lengths both models can be useful. Since the result of the analysis indicated that the gradient length value was shorter than $4 \mathrm{SD}$, a linear method with Principal component analyses (PCA) was considered to be the appropriate. PCA was also preferred because this is a widely used as descriptive data analysis tool, and it is the best technique to use when a dataset approximates to a multivariate normality (Jolliffe \& Cadima, 2016; McCune \& Mefford, 1999). On the other hand, PCA identifying the main axes of variance within a dataset to understand the key variables in the ordination (Jolliffe \& Cadima, 2016). Sampling groups (e.g. studied variables) were analyzed according to: (i) overall, (ii) biophysical and cultural, and (iii) biodiversity. PCA analyses included a Monte Carlo test with 999 permutations to evaluate the significance of each axis. We selected a variance/covariance centered by columns to obtain the cross-products matrix. The calculated scores for the columns were distance-based matrix biplots. Additionally, Multi-Response Permutation 
Procedures (MRPP) were conducted using the Sorensen (Bray-Curtis) distance measures to test statistical differences for all group comparisons (e.g., sample groups). All statistical analyses were performed using PC-Ord software (McCune \& Mefford, 1999).

\section{Results}

Biophysical attributes were the most valued by the visitors (Table 1), mainly from Europe and Central Asia (30\% of frequency). The mountains were the biophysical attribute that was most preferred by the visitors (98\%), although the percentage of selection did not abruptly vary with other attributes such as water, forests and open-lands $(85,83$, and $78 \%$, respectively). Considering the cultural variables, the most observed attribute was the signposts ( $82 \%$ of frequency). There was $15 \%$ less frequency between the most observed attribute (signposts) and the second one (urban areas), and $40 \%$ higher than the other studied attributes such as bays, sights, sailing, etc. Biodiversity had the lower percentage of occurrence regarding to the other two studied attributes. It was possible to determine the species that presented the most relevant occurrence frequency: the native shrubs Chiliotrichum diffusum (13\% of frequency) and Empetrum rubrum (6\%), the native bryophytes and lichens Protousnea spp. (14\%) and Sphagnum magellanicum (7\%), and the native fungi Cyttaria harioti (14\%), as well as the exotic forbs Lupinus polyphyllus (12\%) and Taraxacum officinale (9\%). Regarding the fauna, goose and ducks have the highest frequency of occurrence, mainly because the Magellan goose (Chloephaga picta) had an outstanding occurrence frequency $(>40 \%)$. In addition, this goose was highly valued by Europe and Central Asia, and North America visitors. Similarly, other bird groups as cormorants, wader and penguins $(25,11$, and $11 \%$ of frequency, respectively) were also highly valuated by the visitors. Notably, exotic beavers were the species that most attracted the attention of visitors (44\% occurrence frequency), with a high valuation by Europe and Central Asia (20\%) visitors followed by Latin America and Caribbean (9\%) regions. A similar appreciation occurred with exotic horses (22\% occurrence frequency), which had a high valuation by East Asia and Pacific (7\%), and Latin America and Caribbean (7\%) regions. The eared seals had also an important frequency (24\%), which was recorded by visitors from all regions. This may be due to the existence of specific places for its sighting, which facilitates its observation for all visitors.

Multivariate analyses showed similar trends than those described before (Fig. 4). When overall variables (e.g. biophysical, cultural, and biodiversity) were analysed (Fig. 4a), PCA highlighted the effect of the variable attributes on visitors from the different World's regions. The first three axes explained $22.2 \%(p=0.017), 17.1 \%(p=0.034)$, and $12.7 \%$ $(p=0.623)$ of the variation in the total dataset. Axis 1 was mostly related to biophysical attributes such as mountains (eigenvector $=0.8142$ ) and cultural attributes such as urban landscapes (eigenvector $=-0.3641$ ), sights (eigenvector $=-0.3226$ ), and bays (eigenvector $=-0.1945$ ); while the Axis 2 was more related to biophysical attributes such as water (eigenvector $=-0.7480$ ), forests (eigenvector $=-0.4409$ ), and cultural attributes such as a signposts (eigenvector $=0.1129$ ). The contribution of biodiversity attributes was very low, and not improve the explanation of the plot variation. The specific attributes that most contributed to PCA ordination were the goose and ducks (eigenvector $=0.0756$ ) and eared seals (eigenvector $=-0.0727$ ) for Axis 1 , and rodents (eigenvector $=0.0323$ ) and odd-toed ungulates (eigenvector $=-0.0136$ ) for Axis 2. Notably, the visitors from Europe and Central Asia and North America were mostly related to biophysical variables (e.g. mountains 
A. H. Herrera et al.

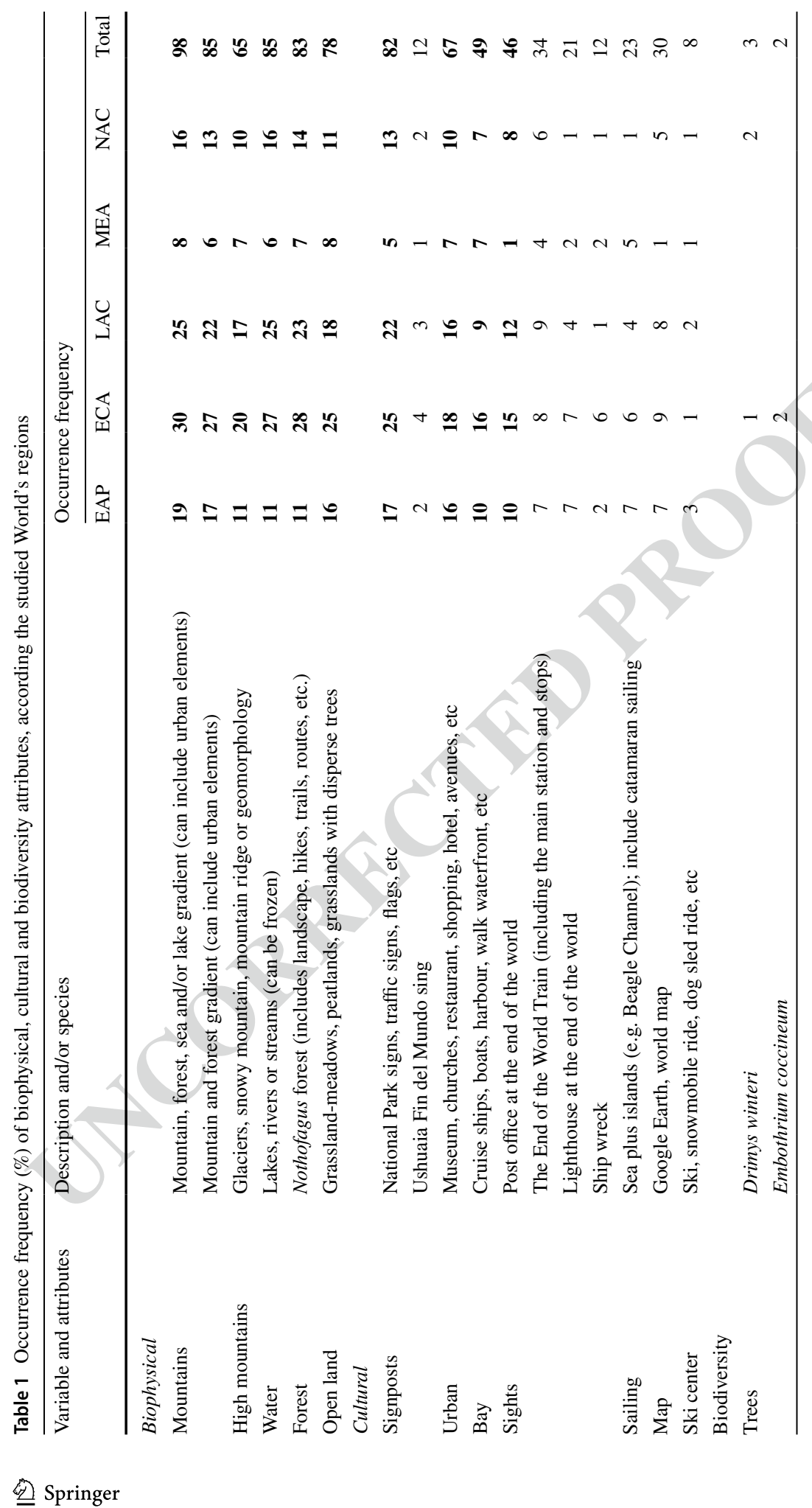




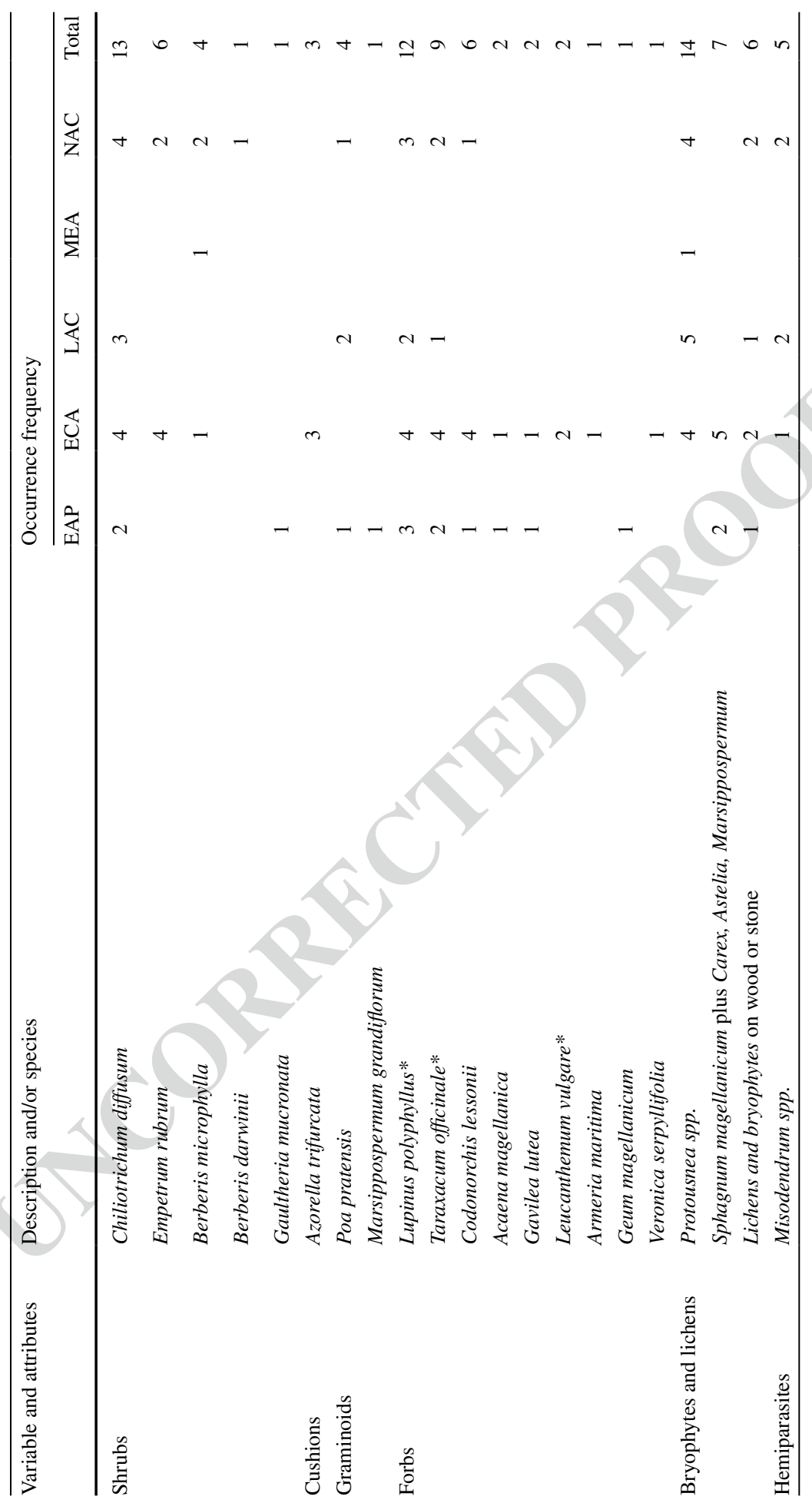




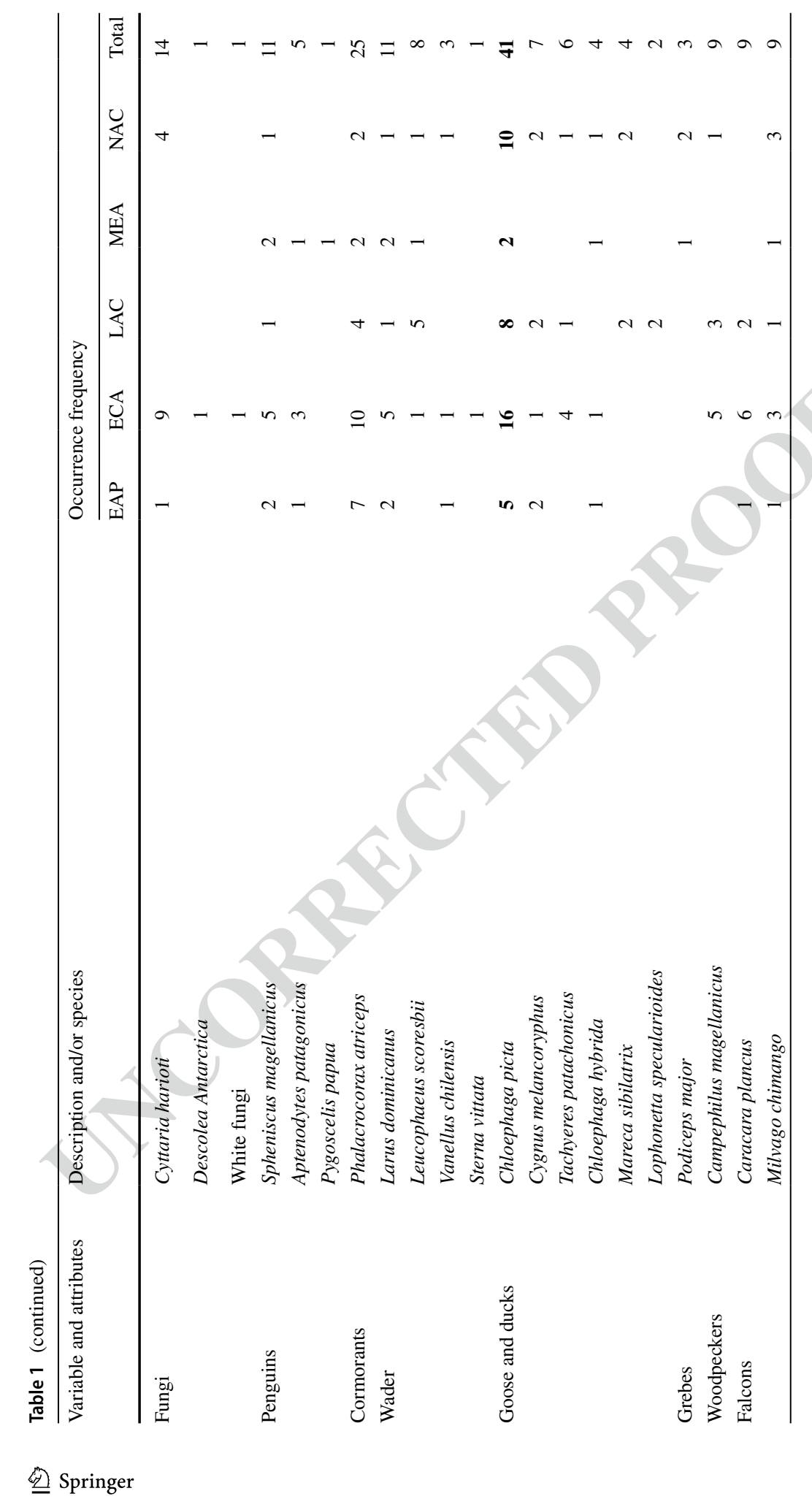

A. H. Herrera et al. 


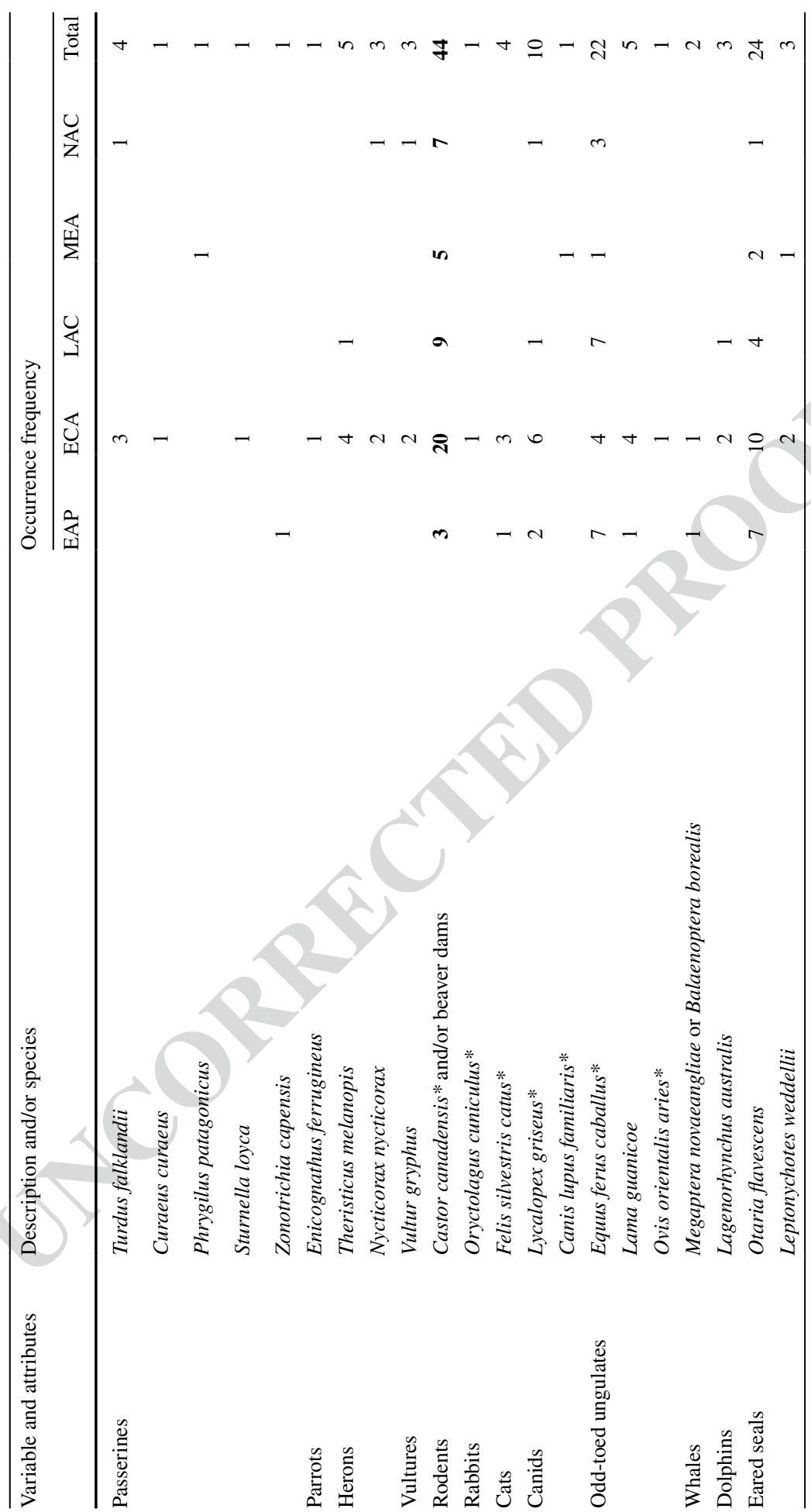



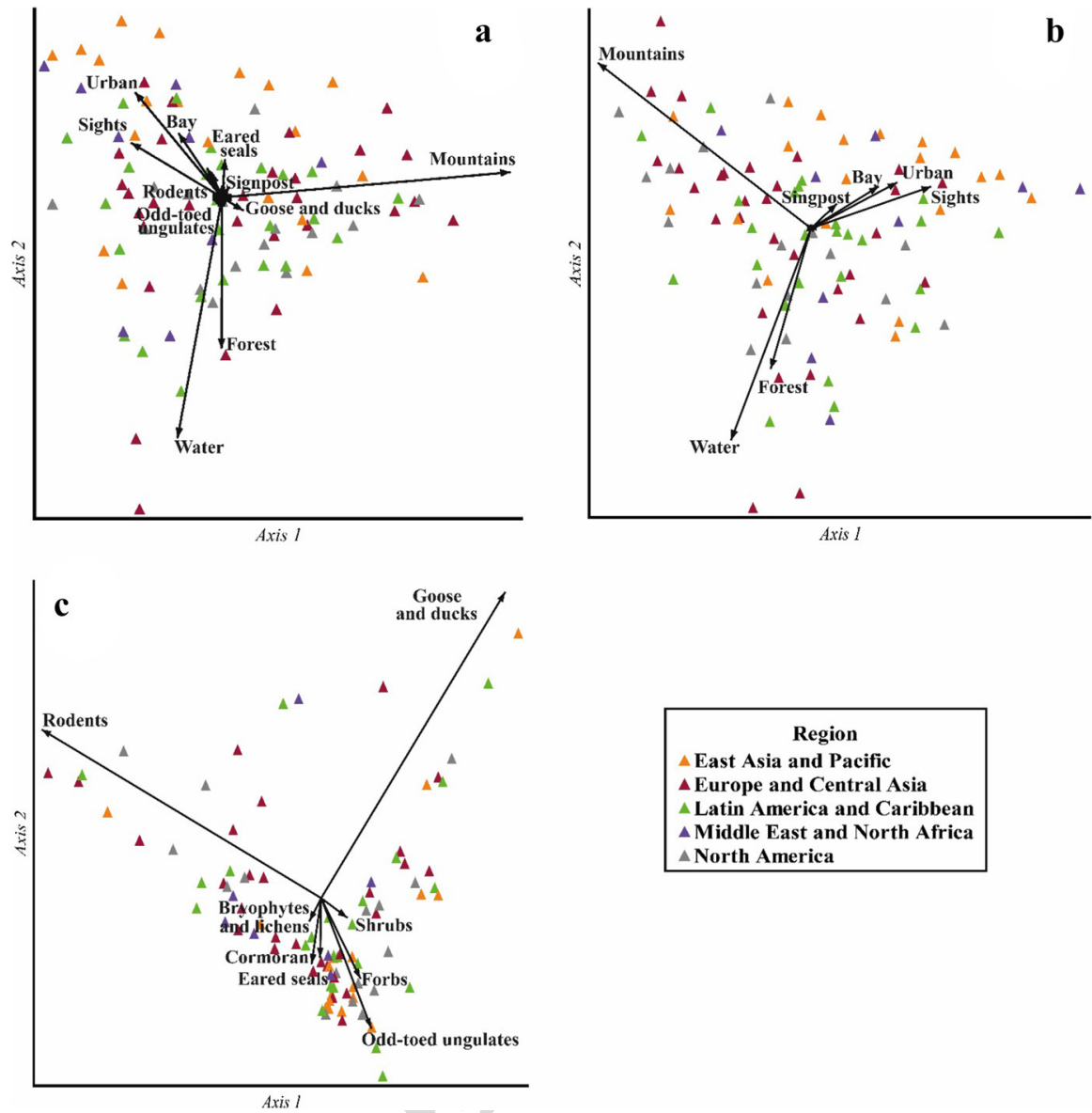

Fig. 4 Principal components analysis (PCA) using the a overall, $\mathbf{b}$ biophysical and cultural, $\mathbf{c}$ and biodiversity analyzed variables. The solid arrows indicate the variable attributes. The attributes that contributed the least to PCA variation were not graphically represented to simplify and clarify the interpretation. Samples were classified (colors) according to the World's regions

and forests) in Axis 1, whereas the visitors from East Asia, Pacific and Middle East, and North Africa were mostly related to cultural variables in Axis 2 (e.g. urban areas), while Latin America and Caribbean visitors can be related to both axes.

When individual PCAs were performed for biophysical and cultural attributes (Fig. 4b), the first three axes explained $33.8 \%(p=0.001), 20.5 \%(p=0.004)$, and $14.1 \%(p=0.440)$ of the variation in the total dataset. The attributes that most contributed in Axis 1 were mountains (eigenvector $=-0.8853$ ) and signposts (eigenvector $=0.0583$ ), while for Axis 2 were water (eigenvector $=-0.7302$ ), forests (eigenvector $=-0.4677$ ), sights (eigenvector $=0.3505$ ), urban areas (eigenvector $=0.2392$ ), and bays (eigenvector $=0.2059$ ). This analyses showed a clear separation between visitors from East Asia and Pacific and the other World's regions for the studied cultural attributes (e.g. urban, sights) in Axis 1, while Europe and Central Asia and North America visitors had a clear separation along the different biophysical attributes (e.g. mountains) in Axis 2. Visitors from Latin America and Caribbean, and Middle East and North Africa were dispersed along both axes. 
When biodiversity attributes were analysed (Fig. 4c), the first three axes explained 19.7\% $(p=0.215), 16.5 \%(p=0.391)$ and $12.4 \%(p=0.379)$ of the total variation of the dataset. For Axis 1 , rodents (eigenvector $=-0.8164$ ), shrubs (eigenvector $=0.0750$ ) and cormorants (eigenvector $=-0.0273$ ) were the attributes that most contributed to the ordination, while ducks (eigenvector $=0.7723$ ), odd-toed ungulates (eigenvector $=-0.3286$ ), eared seals (eigenvector $=-0.1495$ ), bryophytes and lichens (eigenvector $=-0.0571$ ), and forbs (eigenvector $=-0.2023$ ) were the attributes that most contributed in the ordination of Axis 2. Finally, it was observed that the exotic beavers (rodents) and ducks were the attributes that most contributed to the ordination in a whole.

In the MRPP, it was observed significant differences among the regions of visitors considering the biophysical and cultural and biodiversity variables. In overall variables, East Asia and Pacific visitors presented significant differences with other regions (Table 2), e.g. East Asia and Pacific vs. Europe and Central Asia $(\mathrm{T}=-5.270 ; \mathrm{A}=0.028 ; p<0.001)$ and East Asia and Pacific vs. North America $(\mathrm{T}=-4.856 ; \mathrm{A}=0.038 ; p<0.001)$. East Asia and Pacific did not have significant differences with other regions, e.g. Middle East and North Africa $(p=0.233)$. Interestingly, Latin America and Caribbean had significant differences with most of the regions, except with North America $(p=0.655)$. North America and Europe and Central Asia had similar attribute values $(p=0.533)$. Considering the biophysical and cultural variables, the differences were highly marked between the West (Europe and Central Asia, Latin America and Caribbean, and North America) and the East (East Asia and Pacific) regions, e.g. East Asia and Pacific vs. Europe and Central Asia ( $\mathrm{T}=-4.871 ; \mathrm{A}=0.033 ; p<0.001)$; East Asia and Pacific vs. Europe and Central Asia ( $\mathrm{T}=-5.129 ; \mathrm{A}=0.053 ; p<0.001$ ); East Asia and Pacific vs. Latin America and Caribbean $(\mathrm{T}=-4.083 ; \mathrm{A}=0.031 ; p=0.003)$. These differences also occurred between the visitors from Middle East and North Africa, and West regions: Middle East and North Africa vs. Europe and Central Asia ( $\mathrm{T}=-2.677 ; \mathrm{A}=0.024 ; p=0.018)$; Middle East and North Africa vs. North America $(\mathrm{T}=-3.449 ; \mathrm{A}=0.051 ; p=0.006)$; Middle East and North Africa vs. Latin America and Caribbean $(\mathrm{T}=-2.547 ; \mathrm{A}=0.025 ; p=0.019)$. When biodiversity was analysed, there were no significant differences among most of the regions, except between East Asia and Pacific vs. Middle East and North Africa ( $\mathrm{T}=-3.239 ; \mathrm{A}=0.020 ; p=0.008)$, and between Europe and Central Asia, and Latin America and Caribbean $(T=-2.054$; $\mathrm{A}=0.011 ; p=0.039$ ).

\section{Discussion}

\subsection{Biophysical, Cultural and Biodiversity Attributes}

In the studied socio-ecological system, all the identified biophysical and cultural attributes occurred in videos from all the World regions. However, several variables or species selected as biodiversity attributes were absent in videos from Latin America and Caribbean, as well as in videos from Middle East and North Africa (Table 1). Considering the vegetation attributes, high occurrence frequency of both native and exotic species were observed in the biodiversity attributes, probably because these exotic species are conspicuous (e.g. colourful flowers as L. polyphyllus). However, it is interesting to note that other inconspicuous flora (e.g. bryophytes, lichens, and fungi) had a high preference by visitors from Europe and Central Asia, Latin America and Caribbean, and North America. In addition, it is interesting that well-known charismatic species, such as penguins, had a lower 
Assessing Socio-ecological Systems Using Social Media Data:...

Table 2 Multi-Response Permutation Procedures (MRPP) test to evaluate differences among the socio-ecological valuation of visitors from different World's regions (EAP East Asia and Pacific; ECA Europe and Central Asia; $M E A$ Middle East and North Africa; LAC Latin America and Caribbean; NAC North America) according to the overall, biophysical and cultural and biodiversity analyzed attributes

\begin{tabular}{|c|c|c|c|c|}
\hline Level & Group comparison & $T$ & $A$ & $p$ \\
\hline \multirow[t]{10}{*}{ Overall } & EAP vs. MEA & -0.614 & 0.006 & 0.233 \\
\hline & EAP vs. ECA & $-\mathbf{5 . 2 7 0}$ & 0.028 & $<0.001$ \\
\hline & EAP vs. NAC & -4.856 & 0.038 & $<0.001$ \\
\hline & EAP vs. LAC & -4.518 & 0.028 & 0.001 \\
\hline & MEA vs. ECA & -1.480 & 0.010 & 0.084 \\
\hline & MEA vs. NAC & -3.592 & 0.037 & 0.003 \\
\hline & MEA vs. LAC & -3.872 & 0.029 & 0.002 \\
\hline & ECA vs. NAC & 0.233 & -0.001 & 0.533 \\
\hline & ECA vs. LAC & -2.121 & 0.010 & 0.035 \\
\hline & NAC vs. LAC & 0.508 & -0.003 & 0.655 \\
\hline \multirow{10}{*}{$\begin{array}{l}\text { Bio- } \\
\text { physi- } \\
\text { cal and } \\
\text { cul- } \\
\text { tural }\end{array}$} & EAP vs. MEA & 0.538 & -0.007 & 0.660 \\
\hline & EAP vs. ECA & -4.871 & $\mathbf{0 . 0 3 3}$ & $<0.001$ \\
\hline & EAP vs. NAC & -5.129 & 0.053 & $<0.001$ \\
\hline & EAP vs. LAC & -4.083 & 0.031 & 0.003 \\
\hline & MEA vs. ECA & -2.677 & 0.024 & 0.018 \\
\hline & MEA vs. NAC & -3.449 & 0.051 & 0.006 \\
\hline & MEA vs. LAC & -2.547 & 0.025 & 0.019 \\
\hline & ECA vs. NAC & 0.618 & -0.005 & 0.690 \\
\hline & ECA vs. LAC & 0.051 & 0.000 & 0.443 \\
\hline & NAC vs. LAC & 0.360 & -0.003 & 0.580 \\
\hline \multirow{10}{*}{$\begin{array}{l}\text { Biodi- } \\
\text { versity }\end{array}$} & EAP vs. MEA & -1.729 & 0.021 & 0.061 \\
\hline & EAP vs. ECA & $-\mathbf{3 . 2 3 9}$ & 0.020 & 0.008 \\
\hline & EAP vs. NAC & -1.678 & 0.016 & 0.064 \\
\hline & EAP vs. LAC & 0.245 & -0.002 & 0.533 \\
\hline & MEA vs, ECA & 0.303 & -0.002 & 0.551 \\
\hline & MEA vs. NAC & -1.481 & 0.020 & 0.083 \\
\hline & MEA vs. LAC & -0.594 & 0.005 & 0.246 \\
\hline & ECA vs. NAC & -0.618 & 0.004 & 0.231 \\
\hline & ECA vs. LAC & -2.054 & 0.011 & 0.039 \\
\hline & NAC vs. LAC & -0.232 & 0.002 & 0.356 \\
\hline
\end{tabular}

$T$ is the statistic of MRPP, $A$ is the chance-corrected within-group agreement, $p$ is the probability associated with $T$. The significant effects $(p<0.05)$ are printed in bold

289 percentage of selection than goose and ducks. A possible explanation for the higher values 290 of the duck group might be that visitors no need to take a catamaran tour to see them, 291 due to these animals inhabit urban and peri-urban areas with free access. Notably, some 292 exotic species, such as beavers, greatly attracted the attention of visitors. However, this 293 charismatic species causes major alterations to natural ecosystems (e.g. riparian unique 294 forests) disrupting many crucial ecological processes (Henn et al., 2016; Huertas Herrera 295 et al., 2020) and threatening many native species of Tierra del Fuego (Wallem et al., 2010). 296 In this context, the analysis of this social media data also can be linked to environmental 297 policies, whether national or provincial levels, where is necessary to represent the socio298 ecological systems issues covering a major range of topics such as biodiversity and con299 servation connected with public policies (e.g. sense of belonging to nature and cultural 
heritage). Despite accessibility is an important aspect of nature-based tourism demand (Hausmann et al., 2016), managers and conservation authorities should ensure the access to the sense of place to foster the relevance local attributes (e.g. natural and cultural elements) and accordingly, the well-being of the local community. Overall, mammals had a lower occurrence frequency than birds in this study, possibly because some of them are more difficult to observe at these latitudes (e.g. whales with $2 \%$ of frequency). In this context, the entire analysed social media set of attributes can be used to understand the main elements of this socio-ecological system, and it would be possible to find the most relevant ones that require increasing attention of visitors.

\subsection{The Attribute Valuation According to the Visitor Provenance}

The valuation of the biophysical and cultural variables had more differences among visitors from different World's regions than the biodiversity itself. This could be because visitors may spend more time immersed in the biophysical and cultural attributes (e.g. streets, buildings), and as was mentioned above, biodiversity attribute is not always easy to observe at these latitudes. Also, visitors from different regions of the World could differently value the attributes depending on its culture, religious beliefs or age. For this, the different visitors can decide more freely what to film/photograph during more or less time the static attributes. However, the valuation of biodiversity attributes (e.g. species) could be more biased from: (i) existing facilities for approaching to wild areas and for observing their species; (ii) the information about the species (e.g. names, origin, relevance); and (iii) the promotion and dissemination by tourism companies, environmental authorities and local government about certain charismatic or charming species (e.g. specific tours promote beaver observation). As mentioned before, accessibility is an important aspect of nature-based tourism demands (Hausmann et al., 2016). This is an example that a socio-ecological system can be complex to be analysed, where people can have different visions and preferences of the natural and cultural world.

\subsection{Implications of the Studied Approach for Future Investigation}

Our study showed that social media contents, and specifically, screen time lapse can be used as reference to estimate the valuation of biophysical, cultural and biodiversity attributes within one specific socio-ecological system. Previous works have suggested that social media provide data to understand the socio-ecological context perceived by visitors from different regions of the world (Otsuka \& Yamakoshi, 2020; Park et al., 2017). This is likely because many socio-ecological assessments go hand in hand with the human vision of nature and culture, and time is an element that is valued in contemporary culture (e.g. time is money). For Collins et al. (2010) and Lenormand et al. (2018) visitors from different world regions can reveal socio-ecological issues from worldwide (e.g. environmental and ecological crisis, overwhelm by consumerism, technology and industrialization), despite the appreciation of their cultural perception (e.g. cultural phenomenon). That is why the perceived socio-ecological values can be used to better understand human interactions with their natural or urban environment. The world comprises culturing with diverse beliefs and practices (Lenormand et al., 2018), and there may be links between long-distance ecological and social systems that generate conservation actions or policies at a global scale (Newsome \& Hughes, 2018; Rocha et al., 2019). Therefore, it is crucial to understand the link between human 
preferences with distant places from a broad perspective (e.g. global). Our results showed a high value preference for exotic species (e.g. beavers and horses were preferred by visitors from the North Hemisphere).

Traditionally, the method in which information on socio-ecological values are derived from surveys or interviews with different social actors, e.g. the visitor who visits a National Park. Using the interview methods were possible to determine the fundamental information to understand the most relevant human-perception of a place of interest (e.g. how one visitor values the biodiversity in a particular place). However, the visitor answers may be subject to emotions linked to the enthusiasm of visiting one special place (Markham, 2005), and in consequence, it may not reflect what a person really value. In this context, social media data can be useful and/or a complementary method for an objective understanding of the factors that shape human attitudes (Beneito-Montagut, 2011; Toivonen et al., 2019). There is a range of biophysical, cultural and biodiversity attributes that can allowed to identify and analyse the data gathered from social media. In fact, this data allows the intersection of open information (e.g. such as World Bank datasets) on people from regions around the world, to generate an effective understanding of the visitors' assessment of one specific socio-ecological system. In addition, the studied regions are not only a sum of visitors from different countries, these analyses must be more diverse due to include different cultures comprised into them (e.g. languages or religions). This would facilitate articulating the valuations of the visitors with the local people to rethink the human relationships with nature as part of a great global system. Which are, among other things, a great contribution of nature to people (Díaz et al., 2018).

Decision-makers could use social media data to recognize the different socio-ecological attributes to develop conservation strategies and in natural and urban sites with the participation of local social actors (Lacitignola et al., 2007). Thus, the observed social media valuations can build the bases of new management opportunities for decision-makers. Local and international institutions can redirect efforts to make the visitor experience more comfortable, educational or increase the entertaining, generating a greater contribution to the socio-ecological system. Understand the preferences of visitors from different regions to see the attributes offered by the socio-ecological system and think if they are adequate to local requirements, and in this context, can be improved or identify if something is missing. This would allow finding entrepreneurship opportunities with precise and specific data on the attributes of the place. From this, attributes are revealed that are important to visitors, which could be used to understanding the interests and motivations of visitors. For example, in our study area, a socio-ecological system was studied where there is an area with a wealth of cultural attributes (e.g. Ushuaia city) separated from an area with high naturalness (e.g. Tierra del Fuego National Park). Our results showed that visitors from certain regions assume different relationships between culture and nature (e.g. West regions for biophysical and biodiversity, and East region for cultural). This is an input of information that allows finding a new analysis of the valuation of biodiversity to enjoy, and allowed the identification of cultures linked to some biodiversity values according the different areas of the world, presenting them as an opportunity to identify key attributes for present and future management and conservation planning. 


\section{Concluding Remarks}

We used YouTube to collect information regarding the valuation of biophysical, cultural and biodiversity attributes of Ushuaia city and its surrounding forested landscapes by worldwide visitors, as an example of a tool for assessing socio-ecological systems issues (e.g. how people value nature). Our working hypothesis was verified. Visitors differentially value the studied socio-ecological attributes. These differences were significantly marked between the West (Europe and Central Asia, Latin America and Caribbean, and North America) and the East (East Asia and Pacific) regions. Furthermore, visitor pref-AQ2 erences were mostly based on biophysical and cultural attributes. To conclude, biodiversity was not a central preference of visitors worldwide; however, this may be because it can be more difficult to see animal species in wild habitats (e.g. the occasional species), and instead, the biophysical and cultural attributes are static in a certain place. Thus, $\mathbf{A Q 3}$ if the socio-ecological system becomes a representation of the cultural or biophysical space, tourist visitors are more likely to undervalue the biodiversity. We recommend the development of a friendly system of information in socio-ecological systems targeted to visitors interested in native species, the conservation of the natural environment, and the sense of belonging to local and foreign beliefs. In addition, this was one example of the usefulness of social media data widely available (e.g. data mining), being possible to find new easy ways to obtain ecological indicators of interest for conservation, planning and territorial strategy.

\section{Appendix}

See Table 3. 


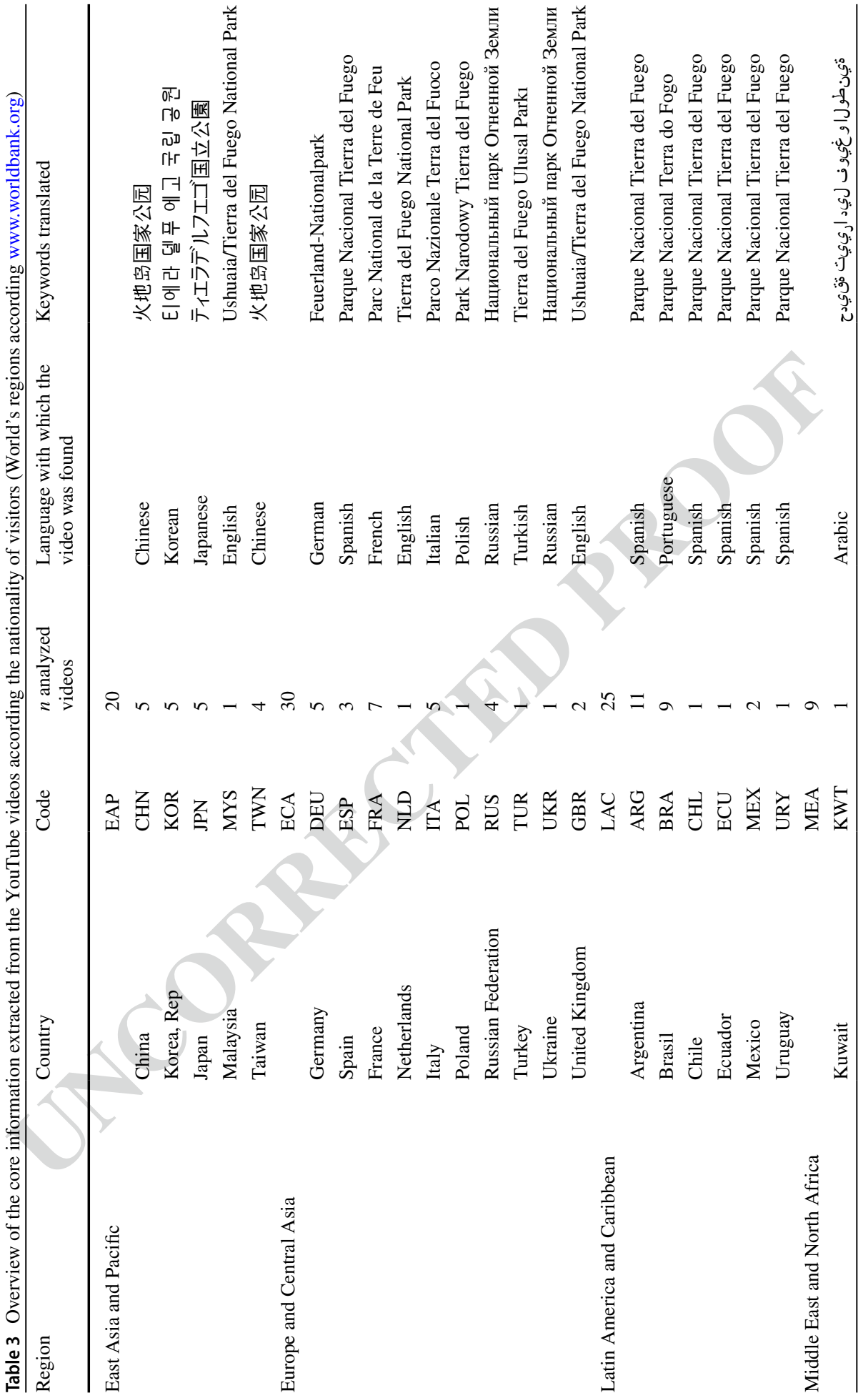




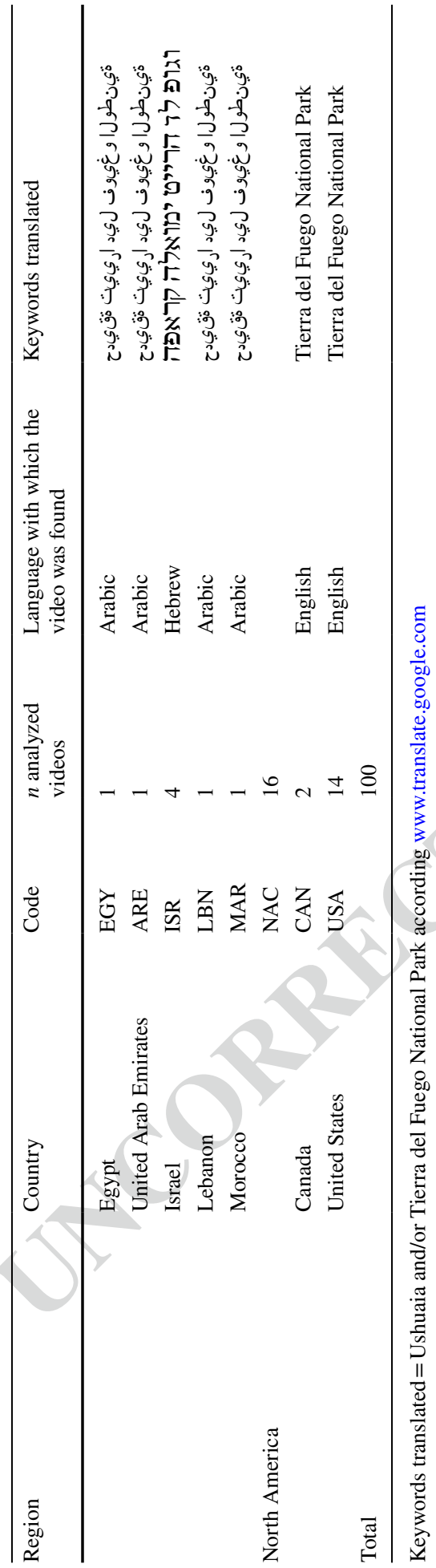


Acknowledgements This work was supported by CONICET postdoctoral scholarships (20192021). We thank the Tierra del Fuego National Park (Administración de Parques Nacionales Permit DRPA/19/2014-2019).

\section{References}

Ballari, S. A., Roulier, C., Nielsen, E. L., Pizarro, J. C., \& Anderson, C. B. (2020). A review of ecological restoration research in the global south and north to promote knowledge dialogue. Conservation and Society, 18, 298-310.

Beneito-Montagut, R. (2011). Ethnography goes online: towards a user-centred methodology to research interpersonal communication on the internet. Qualitative Research, 11, 716-735. https://doi.org/10. $1177 / 1468794111413368$.

Bessi, A., Zollo, F., Del Vicario, M., Puliga, M., Scala, A., Caldarelli, G., Uzzi, B., \& Quattrociocchi, W. (2016). Users polarization on facebook and youtube. PLOS ONE, 11, e0159641. https://doi.org/ 10.1371/journal.pone.0159641.

Burgess, J., \& Green, J. (2018). YouTube: Online video and participatory culture. . Wiley.

Collins, S. L., Carpenter, S. R., Swinton, S. M., Orenstein, D. E., Childers, D. L., Gragson, T. L., Grimm, N. B., Grove, J. M., et al. (2010). An integrated conceptual framework for long-term social-ecological research. Frontiers in Ecology and the Environment, 9, 351-357. https://doi.org/ $10.1890 / 100068$.

Díaz, S., Demissew, S., Carabias, J., Joly, C., Lonsdale, M., Ash, N., Larigauderie, A., Adhikari, J. R., et al. (2015). The IPBES conceptual framework-connecting nature and people. Current Opinion in Environmental Sustainability, 14, 1-16. https://doi.org/10.1016/j.cosust.2014.11.002.

Díaz, S., Pascual, U., Stenseke, M., Martín-López, B., Watson, R. T., Molnár, Z., Hill, R., Chan, K. M., et al. (2018). Assessing nature's contributions to people. Science, 359, 270-272. https://doi.org/10. 1126/science.aap8826.

Fukano, Y., Tanaka, Y., \& Soga, M. (2020). Zoos and animated animals increase public interest in and support for threatened animals. Science of The Total Environment, 704, 135352. https://doi.org/10. 1016/j.scitotenv.2019.135352.

Garavan, T. N., McCarthy, A. M., \& Morley, M. J. (2016). Global human resource development: Regional and country perspectives. . Routledge.

Georgeson, L., Maslin, M., \& Poessinouw, M. (2017). Global disparity in the supply of commercial weather and climate information services. Science Advances, 3, e1602632. https://doi.org/10.1126/ sciadv. 1602632.

Grootjans, A., Iturraspe, R., Lanting, A., Fritz, C., \& Joosten, H. (2010). Ecohydrological features of some contrasting mires in Tierra del Fuego, Argentina. Mires and Peat, 6, 1-15.

Hausmann, A., Slotow, R., Fraser, I., \& Di Minin, E. (2016). Ecotourism marketing alternative to charismatic megafauna can also support biodiversity conservation. Animal Conservation, 20, 91-100. https://doi.org/10.1111/acv.12292.

Henn, J. J., Anderson, C. B., \& Martínez Pastur, G. (2016). Landscape-level impact and habitat factors associated with invasive beaver distribution in Tierra del Fuego. Biological Invasions, 18, 16791688. https://doi.org/10.1007/s10530-016-1110-9.

Hodge, T. (1997). Toward a conceptual framework for assessing progress toward sustainability. Social Indicators Research, 40, 5-98. https://doi.org/10.1023/A:1006847209030.

Huertas Herrera, A., Lencinas, M. V., Toro Manríquez, M., Miller, J. A., \& Martínez Pastur, G. (2020). Mapping the status of the North American beaver invasion in the Tierra del Fuego archipelago. PLOS ONE, 15, e0232057. https://doi.org/10.1371/journal.pone.0232057.

Jolliffe, I. T., \& Cadima, J. (2016). Principal component analysis: A review and recent developments. Philosophical Transactions of the Royal Society A: Mathematical, Physical and Engineering Sciences, 374, 20150202. https://doi.org/10.1098/rsta.2015.0202.

Kim, J. Y., Do, Y., Im, R.-Y., Kim, G.-Y., \& Joo, G.-J. (2014). Use of large web-based data to identify public interest and trends related to endangered species. Biodiversity and Conservation, 23, 29612984. https://doi.org/10.1007/s10531-014-0757-8.

Lacitignola, D., Petrosillo, I., Cataldi, M., \& Zurlini, G. (2007). Modelling socio-ecological tourismbased systems for sustainability. Ecological Modelling, 206, 191-204. https://doi.org/10.1016/j. ecolmodel.2007.03.034. 
Lenormand, M., Luque, S., Langemeyer, J., Tenerelli, P., Zulian, G., Aalders, I., Chivulescu, S., Clemente, P., et al. (2018). Multiscale socio-ecological networks in the age of information. PLoS ONE, 13, e0206672. https://doi.org/10.1371/journal.pone.0206672.

Markham, A. (2005). The methods, politics, and ethics of representation in online ethnography. In N. K. Denzin \& Y. S. Lincoln (Eds.), The SAGE handbook of qualitative methods. (pp. 793-820). SAGE.

Martínez Pastur, G. M., Peri, P. L., Lencinas, M. V., García-Llorente, M., \& Martín-López, B. (2016). Spatial patterns of cultural ecosystem services provision in Southern Patagonia. Landscape Ecology, 31, 383-399. https://doi.org/10.1007/s10980-015-0254-9.

Mastrangelo, M. E., Weyland, F., Herrera, L. P., Villarino, S. H., Barral, M. P., \& Auer, A. D. (2015). Ecosystem services research in contrasting socio-ecological contexts of Argentina: Critical assessment and future directions. Ecosystem Services, 16, 63-73. https://doi.org/10.1016/j.ecoser.2015. 10.001 .

McCune, B., \& M.J. Mefford. (1999). Multivariate analysis of ecological data. Version 4.0. Gleneden Beach: MjM Software design.

Newsome, D., \& Hughes, M. (2018). The contemporary conservation reserve visitor phenomenon! Biodiversity and Conservation, 27, 521-529. https://doi.org/10.1007/s10531-017-1435-4.

Otsuka, R., \& Yamakoshi, G. (2020). Analyzing the popularity of YouTube videos that violate mountain gorilla tourism regulations. PLoS ONE, 15, e0232085. https://doi.org/10.1371/journal.pone.02320 85.

Park, M., Park, J., Baek, Y. M., \& Macy, M. (2017). Cultural values and cross-cultural video consumption on YouTube. PLoS ONE, 12, e0177865. https://doi.org/10.1371/journal.pone.0177865.

Rocha, J. C., Malmborg, K., Gordon, L. J., Brauman, K. A., \& Declerck, F. (2019). Mapping social ecological systems archetypes. Environmental Research Letters, 15, 034017. https://doi.org/10.1088/ 1748-9326/ab666e.

Rosalino, L., Gheler-Costa, C., Santos, G., Gonçalves, M., Fonseca, C., \& Leal, A. (2017). Conservation priorities for elementary school students: Neotropical and European perspectives. Biodiversity and Conservation, 26, 2675-2697. https://doi.org/10.1007/s10531-017-1380-2.

Schröter, M., Kraemer, R., Mantel, M., Kabisch, N., Hecker, S., Richter, A., Neumeier, V., \& Bonn, A. (2017). Citizen science for assessing ecosystem services: Status, challenges and opportunities. Ecosystem Services, 28, 80-94. https://doi.org/10.1016/j.ecoser.2017.09.017.

ter Braak, C. J., \& Šmilauer, P. (2015). Topics in constrained and unconstrained ordination. Plant Ecology, 216, 683-696. https://doi.org/10.1007/s11258-014-0356-5.

Thomas-Walters, L., McNulty, C., \& Veríssimo, D. (2020). A scoping review into the impact of animal imagery on pro-environmental outcomes. Ambio, 49, 1135-1145. https://doi.org/10.1007/ s13280-019-01271-1.

Toivonen, T., Heikinheimo, V., Fink, C., Hausmann, A., Hiippala, T., Järv, O., Tenkanen, H., \& Di Minin, E. (2019). Social media data for conservation science: A methodological overview. Biological Conservation, 233, 298-315. https://doi.org/10.1016/j.biocon.2019.01.023.

Tong, L. C., Acikalin, M. Y., Genevsky, A., Shiv, B., \& Knutson, B. (2020). Brain activity forecasts video engagement in an internet attention market. PNAS, 117, 6936-6941. https://doi.org/10.1073/pnas. 1905178117.

Toro Manríquez, M., Soler, R., Lencinas, M. V., \& Promis, A. (2019). Canopy composition and site are indicative of mineral soil conditions in Patagonian mixed Nothofagus forests. Annals of Forest Science, 76, 117. https://doi.org/10.1007/s13595-019-0886-z.

Wallem, P. K., Anderson, C. B., Martínez Pastur, G., \& Lencinas, M. V. (2010). Using assembly rules to measure the resilience of riparian plant communities to beaver invasion in subantarctic forests. Biological Invasions, 12, 325-335. https://doi.org/10.1007/s10530-009-9625-y.

Publisher's Note Springer Nature remains neutral with regard to jurisdictional claims in published maps and institutional affiliations. 